\title{
Effect of different Fungicide Groups with Insecticides for Control of Foliar Diseases of Onion and their Residue in Bulbs after Harvest
}

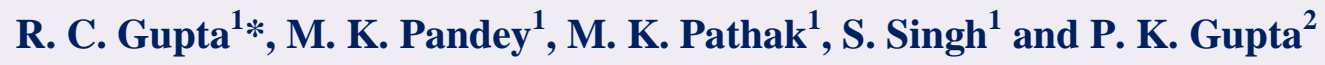 \\ ${ }^{I}$ National Horticultural Research and Development Foundation (NHRDF), \\ Regional Research Station (RRS), Chitegaon phata, Nasihik-Aurangabad Road, \\ Post-Darnasangvi, Taluka-Niphad, Nashik-422 003 (Maharashtra) India \\ ${ }^{2}$ Head Office, Bagwani Bhawan, Janakpuri, New Delhi, India \\ *Corresponding author
}

\section{A B S T R A C T}

\section{Keywords}

Onion,

stemphyllium

blight, purple

blotch, incidence,

intensity, fungicide

group, percent

disease control

(PDC)

Article Info

Accepted:

20 May 2021

Available Online:

10 June 2021
Onion (Allium cepa L.) is one of the important export oriented bulb crop and used as a fresh vegetable, salad and spices. Onion is grown all over the country in which Maharashtra is a leading state occupying the first position in production. Onion crop is affected by various foliar diseases in which stemphylium blight (Stemphylium vesicarium) and purple blotch (Alternaria porri) are the major constraint in bulb production and cause severe yield loss. The field study was carried out with the objectives to evaluate the efficacy of triazole, strobilurin, benzimidazole, dithaiocarbamate and combined fungicides in sequential spray alongwith insecticides for control of foliar diseases of onion and the residue status of pesticide in onion bulbs after harvest of onion. The trial was conducted on onion variety Agrifound Dark Red at Regional Research Station, NHRDF, Nashik, Maharashtra during kharif 2018, and 2019. The pooled data of two years revealed that, the combined spray of different fungicide groups with insecticides Pyraclostrobin+Metiram @ 0.25\% + Deltamethrin @ $0.1 \%$ at 30 DAT, Trifloxistrobin+Tebuconazole @ 0.1\% + Cyperpethrin @ 0.1\% at 45 DAT, Zineb+Hexaconazole @ 0.25\% + Fipronil @ $0.1 \%$ at 60 DAT, Carnebdazim+Mancozeb @ 0.25\%+Curacron@ @ 0.1\% at 75 DAT performed better for management of foliar diseases with percent disease control of stemphylium blight $(61.15 \%)$ and purple blotch $(43.50 \%)$ over untreated control. The onion bulbs were found free from pesticide residue tested after harvesting in NABL accredited Laboratory, NHRDF, Chitegaon, Nashik.

\section{Introduction}

Onion (Allium cepa L.) is an important export oriented vegetable crop in India. It occupies second ranks in area (1219.52 thousand hectares) as well as production (261.0 lakh tonnes) of onion in the country. Maharashtra state occupying the first position with 8047.0 thousand tonnes production of onion from the area of 450 thousand hectares during 2018-19. 
However, the average productivity of onion is $18.71 \mathrm{t} /$ ha in the country which is quite low. Several factors are responsible for low productivity of onion in which the foliar diseases like stemphylium blight (Stemphylium vesicarium), purple blotch (Alternaria porri) are the major threats and causing considerable losses in onion bulb production. Thrips is also a major pest of onion crop causing severe damage to the crop. The yield losses to onion crop due to severe attack of Thrips tabaci was reported to be as high as 90\% (Mote, 1977). The leaves of onion plant infested by thrips (Thrips tabaci L.) are more susceptible to infection by foliar pathogens viz., S. vesicarium and A. porri than uninfested plants and disease severity levels could be higher on onion. Thind and Jhooty (1982) reported the association of thrips with purple blotch infection on onion plants caused by Alternaira porri. Evaluated non-systemic and systemic fungicides against purple blotch of onion caused by $A$. porri under north Indian plains (Priya et al., 2015).

Keeping this in view as above the field study was carried out with the objectives to evaluate the different group of fungicides namely triazole, strobilurin, benzimidazole (systemic), dithaiocarbamate (contact) and combined fungicides (Contact+Systemic) in sequential spray alongwith insecticides for control of foliar diseases of onion and the residue status of pesticide in onion bulbs after harvest of onion.. The trial was conducted during kharif 2018 and 2019 on onion variety Agri found Dark Red at Regional Research Station, NHRDF, Nashik, Maharashtra.

\section{Materials and Methods}

The trial was conducted during kharif 2018 and 2019 on onion variety Agri found Dark Red at Research Farm, NHRDF, Nashik, Maharashtra. Forty five days old seedlings were transplanted at spacing of $15.0 \mathrm{~cm} \mathrm{x}$
$10.0 \mathrm{~cm}$ during the month of August in both the years. The spraying of the different groups of fungicides with insecticide was started after 30 DAT and a total of four sprays were given at 15 days intervals as per treatments. The size of bed was kept as $3.0 \mathrm{~m} \times 1.2 \mathrm{~m}$. The Randomized Block Design was followed with four replications. The standard agronomical practices were followed uniformly in all the treatments. The irrigation was done at regular intervals to maintain the optimum moisture level in soil. The crop was harvested on maturity in month of December during both the years. The data were recorded on incidence and intensity of stemphylium blight and purple blotch before each spray and also the gross and marketable yield of onion bulbs. Disease scoring of foliar disease was done by using 0-5 scale and the intensity or Percent Disease Index (PDI) was drawn as per standard described by Wheeler (1969). The percent disease control and yield increase $(\%)$ over untreated check were also computed. The onion bulbs samples were dried and well cured of three kilogram after harvest from each treatments and untreated control, the bulbs were tested for residue in Pesticide Residue Analysis Laboratory, using Gas chromatophy and tandem mass spectrometry (GC MS/MS) and Liquid chromatophy and tandem mass spectrometry (LC MS/MS). The pesticides were tested by using validated method at recovery ranges between $80 \%$ $120 \%$ with relative standard deviation (RSD) $<10 \%$. The data of two consecutive years of kharif, 2018 and 2019 are presented and also combined data of two years, analyzed statistically and are presented in Tables 1, 2 and 3 for kharif, 2018 and 2019 and combined data of two years respectively. The details of treatments are as follows:

\section{Details of treatments}

$\mathrm{T}_{1^{-}}$Sprays of Propineb @ $0.25 \%+$ Deltamethrin@0.1\% at 30 DAT, Mancozeb 
@0.25\%@0.1\%+Cyperpethrin@0.1\% at 45 DAT, Zineb@0.25\% + Fipronil @ 0.1\% at 60 DAT, Metiram@0.25\%+Curacron@ $0.1 \%$ at 75 DAT

$\mathrm{T}_{2^{-}}$Sprays of Propiconazole @ $0.1 \%+$ Deltamethrin @ $0.1 \%$ at 30 DAT, Epoxiconazole @ 0.1\% + Cyperpethrin @ $0.1 \%$ at 45 DAT, Tricyclazole @ $0.1 \%+$ Fipronil @0.1\% at 60 DAT, Hexaconazole @ $0.1 \%$ + Curacron@ $0.1 \%$ at 75 DAT

$\mathrm{T}_{3^{-}}$Sprays of Pyraclostrobin+Metiram @ $0.25 \%$ + Deltamethrin @ 0.1\% at 30 DAT, Trifloxistrobin+Tebuconazole @ $0.1 \%+$ Cyperpethrin @ 0.1\% at 45 DAT, Zineb+ Hexaconazole@0.25\% + Fipronil @ 0.1\% at 60 DAT, Carnebdazim+Mancozeb @ $0.25 \%+$ +Curacron@ $0.1 \%$ at 75 DAT

$\mathrm{T}_{4^{-}}$Sprays of Propineb @ $0.25 \%+$ Deltamethrin @ $0.1 \%$ at 30 DAT, Propiconazole @ $0.1 \%$ + Cyperpethrin @ $0.1 \%$ at 45 DAT, Pyraclostrobin+Metiram @ $0.25 \%$ + Fipronil @ 0.1\% at 60 DAT, Carnebdazim+Mancozeb @ 0.25\%+Curacron @ $0.1 \%$ at 75 DAT

$\mathrm{T}_{5}$ - Untreated control

\section{Results and Discussion}

\section{(Kharif, 2018)}

The data presented in table 1 revealed that, stemphylium blight disease incidence ranged from 30.0 to $86.67 \%$ with intensity from 1.47 to $14.40 \%$ in different treatments during the cropping period. The lowest and same intensity $(1.47 \%)$ of stemphylium blight was recorded in three treatments $\mathrm{T}_{2}$ (Sprays of Propiconazole @ $0.1 \%+$ Deltamethrin @ $0.1 \%$ at 30 DAT, Epoxiconazole @ 0.1\% + Cyperpethrin @ $0.1 \%$ at 45 DAT, Tricyclazole @ 0.1\% + Fipronil @ 0.1\% at 60 DAT,Hexaconazole@0.1\%+Curacron@
$0.1 \%$ at 75 DAT), $\mathrm{T}_{3}$ (Sprays of Pyraclostrobin+Metiram @ $0.25 \%+$ Deltamethrin @ $0.1 \%$ at 30 DAT, Trifloxistrobin+ Tebuconazole @0.1\% + Cyperpethrin@ $0.1 \%$ at 45 DAT, Zineb+ Hexaconazole @ 0.25\% + Fipronil @ 0.1\% at 60 DAT, Carnebdazim+Mancozeb@0.25\%+ Curacron@0.1\% at 75 DAT) and $\mathrm{T}_{4}$ (Sprays of Propineb @ 0.25\%+Deltamethrin @ 0.1\% at 30 DAT, Propiconazole @ 0.1\% + Cyperpethrin @ $0.1 \%$ at 45 DAT, Pyraclostrobin+Metiram @ 0.25\% + Fipronil @ $0.1 \%$ at 60 DAT, Carnebdazim+Mancozeb @ $0.25 \%+$ Curacron@ $0.1 \%$ at 75 DAT) before second spray at 45 DAT which was at par with other treatments except untreated control. However, the lowest incidence (30.0\%) of stemphylium blight disease was recorded in treatment $\mathrm{T}_{3}$ at $45 \mathrm{DAT}$ and it was at par with $\mathrm{T}_{2}$ and $\mathrm{T}_{4}$.

Further, the lowest intensity $(3.47 \%)$ was recorded in treatment $\mathrm{T}_{3}$ before third spray at 60 DAT and it was at par with $\mathrm{T}_{2}$ and $\mathrm{T}_{4}$, however, the same incidence $(43.33 \%)$ were recorded in $\mathrm{T}_{2}, \mathrm{~T}_{3}$ and $\mathrm{T}_{4}$. The lowest intensity $(4.53 \%)$ was recorded in treatment $\mathrm{T}_{3}$ and $\mathrm{T}_{4}$ before fourth spray at 75 DAT and it was at par with all other treatments except untreated control, however, the same incidence $(50.0 \%)$ were recorded in $\mathrm{T}_{3}$ and $\mathrm{T}_{4}$ during the same period of observations in onion. The highest disease intensity (14.40\%) and incidence $(86.67 \%)$ were recorded in untreated control before fourth spray at 75 DAT.

The data on purple blotch disease indicates that the disease was appeared before fourth spray at 75 DAT in onion with very low intensity. The lowest intensity $(0.67 \%)$ with incidence $(16.67 \%)$ were recorded in treatment $\mathrm{T}_{3}$ and the incidence was at par with $\mathrm{T}_{2}$ and $\mathrm{T}_{4}$ at 75 DAT. The disease intensity and incidence were recorded as $1.73 \%$ and $36.67 \%$, respectively in untreated control before fourth spray at 75 DAT. 


\section{Thrips population}

The thrips data did not differ significantly before first spray at 30 DAT. Further, the lowest thrips was recorded before second spray at 45 DAT (3.03 nymps/plant) and before third spray at 60 DAT $(5.67$ nymps/plant) in treatment $T_{4}$ which was found at par with all other treatments except untreated control.

The lowest thrips (3.4 nymps/plant) was recorded before fourth spray at 75 DAT in treatment $\mathrm{T}_{3}$ which was found at par with treatments $\mathrm{T}_{2}$ and $\mathrm{T}_{4}$. The highest thrips (53.90 nymps/plant) was recorded in untreated control before fourth spray at 75 DAT.

\section{Gross and marketable yield of onion}

The highest gross yield (237.49 q/ha) and marketable yield $(173.79 \mathrm{q} / \mathrm{ha})$ were recorded in $\mathrm{T}_{3}$. The lowest gross yield $(199.07 \mathrm{q} / \mathrm{ha})$ and marketable yield $(125.0 \mathrm{q} / \mathrm{ha})$ were recorded in $\mathrm{T}_{5}$ (untreated control).

\section{Pesticide residue status in onion bulbs}

The bulb were tested by using validated method at recovery ranges between $80 \%$ $120 \%$ with relative standard deviation (RSD) $<10 \%$. No residue of both pesticides were detected in the bulbs of all treatments.

The present investigation revealed that the onion bulbs were free from pesticide residue. The use of tested pesticides alone or in combinations for the management of foliar diseases and thrips of onion during kharif is safe and suitable for consumption as well as export.

\section{(Kharif, 2019)}

The data presented in Table 2 revealed that stemphylium blight disease incidence ranged from 15.0 to $85.0 \%$ with intensity from 0.90 to $16.50 \%$ in different treatments during the entire cropping period.

The lowest intensity $(0.90 \%)$ with incidence (15.0\%) of stemphylium blight were recorded in treatment $\mathrm{T}_{3}$ (Sprays of Pyraclostrobin+ Metiram@0.25\% + Deltamethrin@0.1\% at 30 DAT, Trifloxistrobin + Tebuconazole @ $0.1 \%$ + Cyperpethrin@ $0.1 \%$ at 45 DAT, Zineb+ Hexaconazole @ 0.25\% + Fipronil@ $0.1 \%$ at 60 DAT, Carnebdazim+Mancozeb @ $0.25 \%+$ +Curacron@ @ $0.1 \%$ at 75 DAT) before third spray at 60 DAT.

The highest intensity (4.0\%) and incidence (40.0\%) of stemphylium blight disease was recorded in untreated control at 60 DAT.

Further, the lowest intensity (7.20\%) and incidence $(45.0 \%)$ of stemphylium blight were recorded again in treatment $T_{3}$ before fourth spray at 75 DAT and the intensity was found at par with treatment $\mathrm{T}_{2}$ (Sprays of Propiconazole@0.1\% + Deltamethrin@ @ $0.1 \%$ at 30 DAT, Epoxiconazole @ 0.1\% + Cyperpethrin @ $0.1 \%$ at 45 DAT, Tricyclazole @ 0.1\% + Fipronil @ 0.1\% at 60 DAT, Hexaconazole @ 0.1\% + Curacron@ $0.1 \%$ at $75 \mathrm{DAT}$ ) and $\mathrm{T}_{4}$ (Sprays of Propineb @ 0.25\% + Deltamethrin@ $0.1 \%$ at $30 \mathrm{DAT}$, Propiconazole@0.1\% + Cyperpethrin @ $0.1 \%$ at 45 DAT, Pyraclostrobin+Metiram @ $0.25 \%$ + Fipronil @ $0.1 \%$ at 60 DAT, Carnebdazim+Mancozeb @ 0.25\%+Curacron @ $0.1 \%$ at 75$)$.

The highest disease intensity (16.50\%) and incidence $(85.0 \%)$ were recorded in untreated control before fourth spray at 75 DAT.

The lowest intensity $(0.70 \%)$ and incidence $(12.50 \%)$ of purple blotch disease were also recorded in treatment $\mathrm{T}_{3}$ at $60 \mathrm{DAT}$. 
Table.1 Effect of different fungicide groups with insecticides for control of foliar diseases, thrips and yield of onion during kharif, 2018

\begin{tabular}{|c|c|c|c|c|c|c|c|c|c|c|c|c|c|c|c|c|}
\hline \multirow{4}{*}{ Treatments } & \multicolumn{10}{|c|}{ Incidence $(\%)$ and intensity $(\%)$ of foliar diseases of onion } & \multirow{2}{*}{\multicolumn{4}{|c|}{$\begin{array}{c}\text { Average thrips population } \\
\text { Nymphs/plant }\end{array}$}} & \multicolumn{2}{|c|}{ Bulb yield } \\
\hline & \multicolumn{8}{|c|}{ Stemphylium blight } & \multirow{2}{*}{\multicolumn{2}{|c|}{$\begin{array}{l}\text { Purple blotch } \\
75 \text { DAT }\end{array}$}} & & & & & \multirow{3}{*}{$\begin{array}{l}\text { Gross } \\
\text { (q/ha) }\end{array}$} & \multirow{3}{*}{$\begin{array}{c}\text { Marketable } \\
\text { (q/ha) }\end{array}$} \\
\hline & \multicolumn{2}{|c|}{$30 \mathrm{DAT}$} & \multicolumn{2}{|c|}{$45 \mathrm{DAT}$} & \multicolumn{2}{|c|}{$60 \mathrm{DAT}$} & \multicolumn{2}{|c|}{75 DAT } & & & 30 & 45 & 60 & 75 & & \\
\hline & Inc. & Int. & Inc. & Int. & Inc. & Int. & Inc. & Int. & Inc. & Int. & DAT & DAT & DAT & DAT & & \\
\hline $\mathbf{T}_{1}$ & 0.0 & 0.0 & $\begin{array}{c}43.33 \\
(41.15)\end{array}$ & $\begin{array}{c}1.87 \\
(1.54)\end{array}$ & $\begin{array}{c}53.33 \\
(46.92)\end{array}$ & $\begin{array}{c}4.13 \\
(2.15) \\
\end{array}$ & $\begin{array}{c}63.33 \\
(52.78)\end{array}$ & $\begin{array}{c}4.93 \\
(2.33)\end{array}$ & $\begin{array}{c}26.67 \\
(31.00)\end{array}$ & $\begin{array}{c}3.77 \\
(1.35)\end{array}$ & $\begin{array}{c}7.57 \\
(2.84)\end{array}$ & $\begin{array}{c}3.63 \\
(2.03) \\
\end{array}$ & $\begin{array}{c}6.23 \\
(2.58) \\
\end{array}$ & $\begin{array}{c}4.20 \\
(2.17)\end{array}$ & 212.29 & 146.11 \\
\hline $\mathbf{T}_{2}$ & 0.0 & 0.0 & $\begin{array}{c}33.33 \\
(35.22)\end{array}$ & $\begin{array}{c}1.47 \\
(1.40)\end{array}$ & $\begin{array}{c}43.33 \\
(41.15)\end{array}$ & $\begin{array}{c}4.00 \\
(2.12)\end{array}$ & $\begin{array}{c}60.00 \\
(50.77)\end{array}$ & $\begin{array}{c}4.80 \\
(2.30)\end{array}$ & $\begin{array}{c}27.50 \\
(26.57)\end{array}$ & $\begin{array}{c}4.27 \\
(1.25)\end{array}$ & $\begin{array}{c}7.43 \\
(2.81)\end{array}$ & $\begin{array}{c}3.60 \\
(2.02)\end{array}$ & $\begin{array}{c}6.93 \\
(2.73)\end{array}$ & $\begin{array}{c}3.67 \\
(2.04)\end{array}$ & 214.72 & 161.26 \\
\hline $\mathbf{T}_{3}$ & 0.0 & 0.0 & $\begin{array}{c}30.00 \\
(33.21)\end{array}$ & $\begin{array}{c}1.47 \\
(1.40)\end{array}$ & $\begin{array}{c}43.33 \\
(41.15)\end{array}$ & $\begin{array}{c}3.47 \\
(1.99)\end{array}$ & $\begin{array}{c}50.00 \\
(45.00)\end{array}$ & $\begin{array}{c}4.53 \\
(2.24)\end{array}$ & $\begin{array}{c}26.67 \\
(23.86)\end{array}$ & $\begin{array}{c}3.00 \\
(1.08)\end{array}$ & $\begin{array}{c}7.70 \\
(2.86)\end{array}$ & $\begin{array}{c}3.70 \\
(2.05)\end{array}$ & $\begin{array}{c}6.57 \\
(2.65)\end{array}$ & $\begin{array}{c}3.40 \\
(1.97)\end{array}$ & 237.49 & 173.79 \\
\hline $\mathbf{T}_{4}$ & 0.0 & 0.0 & $\begin{array}{c}33.33 \\
(35.22)\end{array}$ & $\begin{array}{c}1.47 \\
(1.40)\end{array}$ & $\begin{array}{c}43.33 \\
(41.15)\end{array}$ & $\begin{array}{c}4.00 \\
(2.12)\end{array}$ & $\begin{array}{c}45.00 \\
(45.00)\end{array}$ & $\begin{array}{c}4.53 \\
(2.24)\end{array}$ & $\begin{array}{c}26.67 \\
(26.57)\end{array}$ & $\begin{array}{c}3.27 \\
(1.30)\end{array}$ & $\begin{array}{c}7.70 \\
(2.86)\end{array}$ & $\begin{array}{c}3.03 \\
(1.88)\end{array}$ & $\begin{array}{c}5.67 \\
(2.47)\end{array}$ & $\begin{array}{c}3.70 \\
(2.05)\end{array}$ & 215.74 & 152.77 \\
\hline $\mathbf{T}_{5}$ & 0.0 & 0.0 & $\begin{array}{c}50.00 \\
(45.00)\end{array}$ & $\begin{array}{c}2.80 \\
(1.81)\end{array}$ & $\begin{array}{c}76.67 \\
(61.22)\end{array}$ & $\begin{array}{c}8.67 \\
(3.02)\end{array}$ & $\begin{array}{c}86.67 \\
(68.86)\end{array}$ & $\begin{array}{l}14.40 \\
(3.86)\end{array}$ & $\begin{array}{c}36.67 \\
(37.22)\end{array}$ & $\begin{array}{c}5.27 \\
(1.49)\end{array}$ & $\begin{array}{c}8.50 \\
(3.00)\end{array}$ & $\begin{array}{l}10.13 \\
(3.26)\end{array}$ & $\begin{array}{l}41.63 \\
(6.47)\end{array}$ & $\begin{array}{l}53.90 \\
(7.37)\end{array}$ & 199.07 & 125.00 \\
\hline CD at $5 \%$ & - & - & 5.00 & 0.18 & 3.04 & 0.14 & 4.61 & 0.25 & 5.86 & 0.12 & NS & 0.21 & 0.62 & 0.18 & 9.71 & 10.63 \\
\hline
\end{tabular}

Inc.: Incidence, Int.: Intensity, DAT: Days after Transplanting

Table.2 Effect of different fungicide groups with insecticides for control of foliar diseases, thrips and yield of onion during kharif, 2019

\begin{tabular}{|c|c|c|c|c|c|c|c|c|c|c|c|c|c|c|c|c|}
\hline \multirow[t]{4}{*}{ Treatments } & \multicolumn{10}{|c|}{ Incidence (\%) and intensity (\%) of foliar diseases of onion } & \multirow{2}{*}{\multicolumn{4}{|c|}{$\begin{array}{c}\text { Average thrips population } \\
\text { Nymphs/plant }\end{array}$}} & \multicolumn{2}{|c|}{ Bulb yield } \\
\hline & \multicolumn{6}{|c|}{ Stemphylium blight } & \multicolumn{4}{|c|}{ Purple blotch } & & & & & \multirow{3}{*}{$\begin{array}{l}\text { Gross } \\
\text { (q/ha) }\end{array}$} & \multirow{3}{*}{$\begin{array}{c}\text { Marketable } \\
\text { (q/ha) }\end{array}$} \\
\hline & \multicolumn{2}{|c|}{45 DAT } & \multicolumn{2}{|c|}{$60 \mathrm{DAT}$} & \multicolumn{2}{|c|}{75 DAT } & \multicolumn{2}{|c|}{60 DAT } & \multicolumn{2}{|c|}{75 DAT } & \multirow{2}{*}{$\begin{array}{c}30 \\
\text { DAT }\end{array}$} & \multirow{2}{*}{$\begin{array}{l}45 \\
\text { DAT }\end{array}$} & \multirow{2}{*}{$\begin{array}{c}60 \\
\text { DAT }\end{array}$} & \multirow{2}{*}{$\begin{array}{c}75 \\
\text { DAT }\end{array}$} & & \\
\hline & Inc. & Int. & Inc. & Int. & Inc. & Int. & Inc. & Int. & Inc. & Int. & & & & & & \\
\hline $\mathbf{T}_{1}$ & 0.0 & 0.0 & $\begin{array}{c}35.00 \\
(36.22)\end{array}$ & $\begin{array}{c}3.00 \\
(1.87)\end{array}$ & $\begin{array}{c}72.50 \\
(58.45)\end{array}$ & $\begin{array}{l}14.10 \\
(3.82)\end{array}$ & $\begin{array}{c}27.50 \\
(31.55)\end{array}$ & $\begin{array}{l}1.90 \\
(1.54)\end{array}$ & $\begin{array}{c}60.00 \\
(50.83)\end{array}$ & $\begin{array}{c}7.00 \\
(2.73)\end{array}$ & $\begin{array}{c}3.78 \\
(2.07)\end{array}$ & $\begin{array}{l}13.35 \\
(3.72)\end{array}$ & $\begin{array}{l}3.95 \\
(2.10)\end{array}$ & $\begin{array}{c}0.50 \\
(0.99)\end{array}$ & 150.41 & 104.30 \\
\hline $\mathbf{T}_{2}$ & 0.0 & 0.0 & $\begin{array}{c}30.00 \\
(33.05)\end{array}$ & $\begin{array}{c}2.30 \\
(1.67)\end{array}$ & $\begin{array}{c}57.50 \\
(49.33)\end{array}$ & $\begin{array}{c}8.60 \\
(3.01)\end{array}$ & $\begin{array}{c}25.00 \\
(29.89)\end{array}$ & $\begin{array}{c}1.70 \\
(1.48)\end{array}$ & $\begin{array}{c}45.00 \\
(42.12)\end{array}$ & $\begin{array}{c}6.80 \\
(2.70)\end{array}$ & $\begin{array}{c}3.88 \\
(2.09)\end{array}$ & $\begin{array}{l}13.68 \\
(3.76)\end{array}$ & $\begin{array}{c}3.08 \\
(1.89)\end{array}$ & $\begin{array}{c}0.28 \\
(0.88)\end{array}$ & 159.58 & 113.89 \\
\hline $\mathbf{T}_{\mathbf{3}}$ & 0.0 & 0.0 & $\begin{array}{c}15.00 \\
(22.50)\end{array}$ & $\begin{array}{c}0.90 \\
(1.17)\end{array}$ & $\begin{array}{c}45.00 \\
(42.12)\end{array}$ & $\begin{array}{c}7.20 \\
(2.77)\end{array}$ & $\begin{array}{c}12.50 \\
(20.47)\end{array}$ & $\begin{array}{c}0.70 \\
(1.09)\end{array}$ & $\begin{array}{c}32.50 \\
(34.72)\end{array}$ & $\begin{array}{c}5.20 \\
(2.39)\end{array}$ & $\begin{array}{c}3.33 \\
(1.95)\end{array}$ & $\begin{array}{l}12.83 \\
(3.65)\end{array}$ & $\begin{array}{c}3.78 \\
(2.06)\end{array}$ & $\begin{array}{c}0.35 \\
(0.92)\end{array}$ & 178.37 & 123.40 \\
\hline $\mathbf{T}_{4}$ & 0.0 & 0.0 & $\begin{array}{c}30.00 \\
(33.05)\end{array}$ & $\begin{array}{c}1.60 \\
(1.45)\end{array}$ & $\begin{array}{c}55.00 \\
(47.88)\end{array}$ & $\begin{array}{c}7.90 \\
(2.89)\end{array}$ & $\begin{array}{c}22.50 \\
(28.23)\end{array}$ & $\begin{array}{c}1.20 \\
(1.30)\end{array}$ & $\begin{array}{c}42.50 \\
(40.67)\end{array}$ & $\begin{array}{c}6.50 \\
(2.64)\end{array}$ & $\begin{array}{c}3.45 \\
(1.98)\end{array}$ & $\begin{array}{l}13.23 \\
(3.70)\end{array}$ & $\begin{array}{c}3.43 \\
(1.98)\end{array}$ & $\begin{array}{c}0.50 \\
(1.00)\end{array}$ & 165.48 & 116.86 \\
\hline $\mathbf{T}_{5}$ & 0.0 & 0.0 & $\begin{array}{c}40.00 \\
(39.23)\end{array}$ & $\begin{array}{c}4.00 \\
(2.12)\end{array}$ & $\begin{array}{c}85.00 \\
(67.50)\end{array}$ & $\begin{array}{l}16.50 \\
(4.12)\end{array}$ & $\begin{array}{c}35.00 \\
(36.22)\end{array}$ & $\begin{array}{c}2.70 \\
(1.78)\end{array}$ & $\begin{array}{c}65.00 \\
(53.78)\end{array}$ & $\begin{array}{c}8.80 \\
(3.05)\end{array}$ & $\begin{array}{c}6.23 \\
(2.59)\end{array}$ & $\begin{array}{l}24.48 \\
(5.00)\end{array}$ & $\begin{array}{l}10.85 \\
(3.37)\end{array}$ & $\begin{array}{c}9.70 \\
(3.19)\end{array}$ & 124.79 & 91.66 \\
\hline CD at $5 \%$ & - & - & 5.75 & 0.17 & 5.71 & 0.24 & 5.82 & 0.26 & 5.58 & 0.20 & 0.20 & 0.26 & 0.24 & 0.20 & 11.35 & 7.30 \\
\hline
\end{tabular}

Inc.: Incidence, Int.: Intensity, DAT: Days after Transplanting 
Table.3 Effect of different fungicide groups with insecticides for control of foliar diseases, thrips and yield of onion (Combined data kharif, 2018 and 2019)

\begin{tabular}{|c|c|c|c|c|c|c|c|c|c|c|c|c|c|c|c|}
\hline \multirow[t]{4}{*}{ Treatments } & \multicolumn{8}{|c|}{ Incidence (\%) and intensity (\%) of foliar diseases of onion } & \multirow{2}{*}{\multicolumn{4}{|c|}{$\begin{array}{c}\text { Average thrips population } \\
\text { Nymphs/plant }\end{array}$}} & \multicolumn{2}{|c|}{ Bulb yield } & \multirow{4}{*}{$\begin{array}{l}\mathrm{B}: \mathrm{C} \\
\text { ratio }\end{array}$} \\
\hline & \multicolumn{6}{|c|}{ Stemphylium blight } & \multirow{2}{*}{\multicolumn{2}{|c|}{$\begin{array}{l}\text { Purple blotch } \\
75 \text { DAT }\end{array}$}} & & & & & Gross & Mark- & \\
\hline & \multicolumn{2}{|c|}{$45 \mathrm{DAT}$} & \multicolumn{2}{|c|}{$60 \mathrm{DAT}$} & \multicolumn{2}{|c|}{75 DAT } & & & \multirow{2}{*}{$\begin{array}{c}30 \\
\text { DAT }\end{array}$} & \multirow{2}{*}{$\begin{array}{c}45 \\
\text { DAT }\end{array}$} & \multirow{2}{*}{$\begin{array}{c}60 \\
\text { DAT }\end{array}$} & \multirow{2}{*}{$\begin{array}{c}75 \\
\text { DAT }\end{array}$} & \multirow[t]{2}{*}{ (q/ha) } & \multirow[t]{2}{*}{ (q/ha) } & \\
\hline & Inc. & Int. & Inc. & Int. & Inc. & Int. & Inc. & Int. & & & & & & & \\
\hline $\mathbf{T}_{1}$ & $\begin{array}{c}18.57 \\
(18.04)\end{array}$ & $\begin{array}{c}0.80 \\
(1.06)\end{array}$ & $\begin{array}{c}42.86 \\
(40.81)\end{array}$ & $\begin{array}{c}3.49 \\
(1.99)\end{array}$ & $\begin{array}{c}68.57 \\
(52.78)\end{array}$ & $\begin{array}{l}10.71 \\
(1.18)\end{array}$ & $\begin{array}{c}45.71 \\
(42.33)\end{array}$ & $\begin{array}{c}4.57 \\
(2.14)\end{array}$ & $\begin{array}{c}5.40 \\
(2.40)\end{array}$ & $\begin{array}{c}9.19 \\
(3.00)\end{array}$ & $\begin{array}{c}4.93 \\
(2.31)\end{array}$ & $\begin{array}{c}2.09 \\
(1.49)\end{array}$ & 176.93 & 122.22 & $5.45: 1$ \\
\hline $\mathbf{T}_{\mathbf{2}}$ & $\begin{array}{c}14.29 \\
(15.50)\end{array}$ & $\begin{array}{c}0.63 \\
(1.01)\end{array}$ & $\begin{array}{c}35.71 \\
(36.53)\end{array}$ & $\begin{array}{c}3.03 \\
(1.86)\end{array}$ & $\begin{array}{c}58.57 \\
(50.77)\end{array}$ & $\begin{array}{c}6.97 \\
(2.71)\end{array}$ & $\begin{array}{c}34.29 \\
(35.45)\end{array}$ & $\begin{array}{c}4.34 \\
(2.08)\end{array}$ & $\begin{array}{c}5.40 \\
(2.40)\end{array}$ & $\begin{array}{c}9.36 \\
(3.01)\end{array}$ & $\begin{array}{c}4.73 \\
(2.24)\end{array}$ & $\begin{array}{c}1.73 \\
(1.37)\end{array}$ & 183.21 & 134.19 & 11.88:1 \\
\hline $\mathbf{T}_{3}$ & $\begin{array}{c}12.86 \\
(14.64)\end{array}$ & $\begin{array}{c}0.63 \\
(1.01)\end{array}$ & $\begin{array}{c}27.14 \\
(30.49)\end{array}$ & $\begin{array}{c}2.00 \\
(1.52)\end{array}$ & $\begin{array}{c}47.14 \\
(45.00)\end{array}$ & $\begin{array}{c}6.06 \\
(2.54)\end{array}$ & $\begin{array}{c}25.71 \\
(30.06)\end{array}$ & $\begin{array}{c}3.26 \\
(1.83)\end{array}$ & $\begin{array}{c}5.20 \\
(2.35)\end{array}$ & $\begin{array}{c}8.91 \\
(2.96)\end{array}$ & $\begin{array}{c}4.97 \\
(2.31)\end{array}$ & $\begin{array}{c}1.66 \\
(1.37)\end{array}$ & 203.71 & 144.99 & $9.21: 1$ \\
\hline $\mathbf{T}_{4}$ & $\begin{array}{c}14.29 \\
(15.50)\end{array}$ & $\begin{array}{c}0.63 \\
(1.01)\end{array}$ & $\begin{array}{c}35.71 \\
(36.53)\end{array}$ & $\begin{array}{c}2.63 \\
(1.73)\end{array}$ & $\begin{array}{c}52.86 \\
(46.65)\end{array}$ & $\begin{array}{c}6.46 \\
(2.61)\end{array}$ & $\begin{array}{c}32.86 \\
(34.63)\end{array}$ & $\begin{array}{c}4.23 \\
(2.07)\end{array}$ & $\begin{array}{c}5.27 \\
(2.36)\end{array}$ & $\begin{array}{c}8.86 \\
(2.92)\end{array}$ & $\begin{array}{c}4.39 \\
(2.19)\end{array}$ & $\begin{array}{c}1.87 \\
(1.45)\end{array}$ & 187.02 & 132.25 & $7.40: 1$ \\
\hline $\mathbf{T}_{5}$ & $\begin{array}{c}21.43 \\
(19.69)\end{array}$ & $\begin{array}{c}1.20 \\
(1.18)\end{array}$ & $\begin{array}{c}55.71 \\
(48.65)\end{array}$ & $\begin{array}{c}6.00 \\
(2.51)\end{array}$ & $\begin{array}{c}85.71 \\
(68.08)\end{array}$ & $\begin{array}{l}15.60 \\
(4.01)\end{array}$ & $\begin{array}{c}52.86 \\
(46.68)\end{array}$ & $\begin{array}{c}5.77 \\
(2.38)\end{array}$ & $\begin{array}{c}7.20 \\
(2.77)\end{array}$ & $\begin{array}{l}18.33 \\
(4.25)\end{array}$ & $\begin{array}{l}24.04 \\
(4.70)\end{array}$ & $\begin{array}{l}28.64 \\
(4.98)\end{array}$ & 156.62 & 105.95 & - \\
\hline CD at $5 \%$ & 1.71 & 1.71 & 3.12 & 0.30 & 3.31 & 0.15 & 3.47 & 0.11 & 0.12 & 0.15 & 0.24 & 0.12 & 6.67 & 10.25 & - \\
\hline
\end{tabular}

Inc.: Incidence, Int.: Intensity, DAT: Days after Transplanting 
The highest intensity and incidence were recorded as $2.70 \%$ and $35.0 \%$, respectively in untreated control before third spray at 60 DAT.

Further, the lowest intensity (5.20\%) and incidence $(32.50 \%)$ at 75 DAT were recorded in treatment $\mathrm{T}_{3}$ before fourth spray. The highest disease intensity and incidence in untreated control before fourth spray at 75 DAT were recorded as $8.80 \%$ and $65.0 \%$, respectively.

\section{Thrips population}

The lowest thrips at 30 DAT (3.33 nymps/plant), 45 DAT (12.83 nymps/plant) were recorded in treatment $\mathrm{T}_{3}$, while lowest thrips at 60 DAT (3.08 nymps/plant) as well as 75 DAT (0.28 nymps/plant) were noted in treatment $T_{2}$ which was found at par with all other treatments except untreated control. The highest thrips (9.70 nymps/plant) were recorded in untreated control before fourth spray at 75 DAT.

\section{Gross and marketable yield of onion}

The highest gross yield (178.37 q/ha) and marketable yield $(123.40 \mathrm{q} / \mathrm{ha})$ were recorded in treatment $\mathrm{T}_{3}$. The lowest gross yield (124.79 q/ha) and marketable yield (91.66 $\mathrm{q} / \mathrm{ha}$ ) were recorded in treatment $\mathrm{T}_{5}$ (untreated control).

\section{Pesticide residue status in onion bulbs}

The dried bulb were tested by using validated method at recovery ranges between $80 \%$ $120 \%$ with relative standard deviation (RSD) $<10 \%$. No residue of both pesticides were detected in the bulbs of all treatments.

The present investigation revealed that the onion bulbs were free from pesticide residue. The use of tested pesticides alone or in combinations for the management of foliar diseases and thrips of onion during kharif is safe and suitable for consumption as well as export.

\section{Combined data (Kharif, 2018 and 2019)}

\section{Effect of treatments on foliar diseases}

The combined data presented in Table 3 revealed that stemphylium blight disease incidence ranged from.0 to $85.71 \%$ with intensity from 0 to $16.60 \%$ in different treatments during the entire cropping period. The lowest intensity with incidence of stemphylium blight were recorded during all the observations in treatment $\mathrm{T}_{3}$ (Sprays of Pyraclostrobin+Metiram @ $0.25 \%+$ Deltamethrin @ $0.1 \%$ at 30 DAT, Trifloxistrobin+Tebuconazole @ $0.1 \%$ + Cyperpethrin @ $0.1 \%$ at 45 DAT, Zineb+ Hexaconazole@0.25\% + Fipronil @0.1\% at 60 DAT, Carnebdazim+Mancozeb@0.25\%+ Curacron@0.1\% at 75 DAT).

The lowest intensity (6.06\%) and incidence $(47.14 \%)$ of stemphylium blight were recorded in treatment $T_{3}$ before fourth spray at 75 DAT and it was found at par with treatment $\mathrm{T}_{4}$. The highest disease intensity $(16.60 \%)$ and incidence $(85.71 \%)$ were recorded in untreated control before fourth spray at 75 DAT.

The lowest intensity (3.26\%) and incidence $(25.71 \%)$ at 75 DAT were recorded in treatment $\mathrm{T}_{3}$ before fourth spray. The highest disease intensity and incidence in untreated control before fourth spray at 75 DAT were recorded as $5.77 \%$ and $52.86 \%$, respectively.

\section{Percent Disease Control (PDC)}

The highest percent disease control (PDC) of stemphylium blight $(61.17 \%)$ and purple blotch $(43.56 \%)$ were recorded in treatment $\mathrm{T}_{3}$ over untreated control at 75 DAT. 


\section{Thrips population}

The lowest thrips at 30 DAT $(5.20$ nymps/plant), 75 DAT (1.66 nymps/plant) were recorded in treatment $T_{3}$, while lowest thrips at 45 DAT (8.86 nymps/plant) as well as 60 DAT (4.39 nymps/plant) were noted in treatment $\mathrm{T}_{4}$ which was found at par with all other treatments except untreated control. The highest thrips (28.64 nymps/plant) were recorded in untreated control before fourth spray at 75 DAT.

\section{Gross and marketable yield of onion}

The highest gross yield (203.71 q/ha) and marketable yield (144.99 q/ha) were recorded in treatment $\mathrm{T}_{3}$. The lowest gross yield $(156.62 \mathrm{q} / \mathrm{ha})$ and marketable yield (105.95 $\mathrm{q} / \mathrm{ha}$ ) were recorded in treatment $\mathrm{T}_{5}$ (untreated control). The higher benefit cost ratio $(11.88: 1)$ was recorded in $T_{2}$ followed by $T_{3}$ (9.21:1) and $\mathrm{T}_{4}(7.40: 1)$.

\section{Pesticide residue status in onion bulbs}

The onion bulbs samples tested during both the kharif trial, 2018 and 2019 in Pesticides Residue Analysis Laboratory, NHRDF, Nashik revealed that no pesticide residue was found in any onion bulb samples after harvest in any treatment including untreated control.

\section{References}

Borkar, S. G., and Patil, B. S. (1995). Chemical control of purple blotch of onion. J. Mycol. Pl. Pathol. 25 (3): 289-290.

Gupta, R. C., and Pandey, N. K. (2011). Integrated disease management for the production of export quality onion bulbs (Allium cepa L.). (Abstr.). National Symposium on Alliums: Current Scenario and Emerging Trends held on March, 12-14 organized by DOGR, Pune,236pp.

Gupta, R. C., Yadav, S. P., and Srivastava, K. J. (2011). Studies on management of foliar diseases of onion (Allium cepa L.) through recommended pesticides and their residue status after harvest. (Abstr.). National Symposium on Alliums: Current scenario and Emerging Trends held on March, 1214 organized by DOGR, Pune, 234$235 \mathrm{pp}$.

Gupta, R. P., and Srivastava, P. K., (1993). Studies on the intervals and quantity of solution of mancozeb for mixed pesticides on the control of diseases and thrips insects pests of Kharif onion. AADF News Letter, 13 (1):1214.

Gupta, R. P., Srivastava, P. K., and Sharma, R. C. (1996). Efficacy of fungicides and their spray intervals on the control of purple blotch and stemphyllium blight disease of onion. NHRDF Newsletter, 16 (4):11-13.

Mathur, K., and Sharma, S. N. (2006). Evaluation of fungicides against Alternaria porri and Stemphyllium vesicarium disease of onion in Rajasthan. J. Mycol. Pl. Pathol., 36(2):323-324.

Mote, U. N. (1977). Efficacy of different insecticides against onion thrips (Thrips tabaci). Maharashtra Agril. University, 2 (1):69-70.

Priya, R. U., Sataraddi, A. and Darshan, S. (2015). Efficacy of non-systemic and systemic fungicides against purple blotch of onion (Allium cepa L.) caused by Alternaria porri (Ellis) Cif.. Int. J. Recent Scientific Res., 6:65196521.

Thind, T. S. and Jhooty, J. S. (1982). Association of thrips with purple blotch infection on onion plants caused by Alternaira porri. Indian Phytopath., 35:696-698. 
Vijaya, M and Rahman, M. A. (2004). Wheeler, B. E. J. (1969). An Introduction to Efficacy of fungicides in control of leaf blight disease of onion (Allium cepa L.). J. Mycol. Pl. Pathol., 34 (2): 654-655. Plant Diseases, First Edition, John wiley \& Sons Ltd., London, UK, pp 301.

\section{How to cite this article:}

Gupta, R. C., M. K. Pandey, M. K. Pathak, S. Singh and Gupta, P. K. 2021. Effect of different Fungicide Groups with Insecticides for Control of Foliar Diseases of Onion and their Residue in Bulbs after Harvest. Int.J.Curr.Microbiol.App.Sci. 10(06): 802-810.

doi: https://doi.org/10.20546/ijcmas.2021.1006.085 